\title{
Avaliação do Uso de Cateter Urinário e Fatores Relacionados em Unidade de Terapia Intensiva Neonatal*
}

\author{
Evaluation of the Use of Urinary Catheter and Related Factors in Neonatal \\ Intensive Care Unit
}

\section{Evaluación del uso de Catéter Urinario y Factores Relacionados en Unidad de Terapia Intensiva Neonatal}

\author{
Joyce Silva dos Santos', Ivone Kamada², Pedro Sadi³, Cristine Alves Costa de Jesus ${ }^{4}$
}

\begin{abstract}
RESUMO
Estudo quantitativo transversal para se verificar a prevalência de cateterização urinária bem como caracterizar os recém-nascidos (RNs) internados em Unidade de Terapia Intensiva (UTI) Neonatal e que necessitam desse procedimento. Foram avaliados prontuários de 128 RNs admitidos entre janeiro de 2008 e dezembro de 2010 na UTI Neonatal de um hospital do Distrito Federal especializado em parto de alto risco. Os dados coletados foram analisados por meio do programa Statistical Package for the Social Sciences (SPSS) 11.5 for Windows $®$. Foi utilizado o teste do $\chi^{2}$ de Mantel-Haenszel, com intervalo de confiança de 95\%, e o teste t de Student, sendo considerados valores significantes $p<0,05$. Foi encontrada prevalência de uso de cateter urinário de 15,6\%. O principal motivo para a indicação do cateter foi a oligúria, com o objetivo de controle do débito urinário. Cateteres urinários em UTI Neonatal estão associados à maior morbidade relacionada a um maior risco para sepse e óbito.
\end{abstract}

DESCRITORES: Cateterismo urinário. Recém-nascido. Prevalência. Enfermagem neonatal.

\begin{abstract}
Transversal and quantitative study to verify the prevalence of urinary catheterization and to characterize the newborn babies (NBs) interned in Neonatal Intensive Care Unit (ICU) and who need this procedure. Medical records of 128 NBs admitted between January 2008 and December 2010 in the Neonatal ICU of a hospital of the Brazilian Federal District, specialized in high-risk childbirth, were evaluated. The data were analyzed through Statistical Package for the Social Sciences (SPSS) 11.5 for Windows ${ }^{\circledR}$. The Mantel-Haenszel $\chi^{2}$ test, with confidence interval of 95\%, and Student's t-test were used, being significant p-values $<0.05$. It was found urinary catheter's prevalence of $15.6 \%$. The main reason for the indication of the catheter was oliguria, with the goal of controlling the urinary debit. Urinary catheters in Neonatal ICU are associated to a higher morbidity related to a higher risk for sepsis and death.
\end{abstract}

DESCRIPTORS: Urinary catheterization. Infant, newborn. Prevalence. Neonatal nursing.

\footnotetext{
${ }^{1}$ Enfermeira na Unidade Neonatal do Hospital das Forças Armadas (HFA). Aluna do Curso de Especialização Enfermagem em Estomaterapia da Universidade de Brasília (UnB). Endereço para correspondência: Setor de Habitações Individuais Norte (SHIN), Centro de Atividades (CA) 05, Bloco C3, Edifício Firenze, apto. 312 - CEP: 71503505 - Lago Norte - Brasília (DF), Brasil - E-mail: jsd.enf@gmail.com

²Professora Adjunto do Departamento de Enfermagem da UnB - Brasília (DF), Brasil. Doutora em Enfermagem pela Universidade de São Paulo (USP) São Paulo (SP), Brasil.

${ }^{3}$ Professor Adjunto do Departamento de Enfermagem da UnB - Brasília (DF), Brasil. Doutor em Ciências da Saúde pela UnB - Brasília, DF. Pós-doutor pela University College of London - Londres, Reino Unido.

${ }^{4}$ Professora Adjunto do Departamento de Enfermagem da UnB - Brasília (DF), Brasil. Doutora em Enfermagem Fundamental pela USP - São Paulo (SP), Brasil. Membro da American Nursing Diagnosis Association (NANDA- I) - Kaukauna (WI), Estados Unidos.

*Artigo extraído de monografia de mesmo título referente à conclusão do curso de especialização de Enfermagem em Estomaterapia pela

Universidade de Brasília, área de concentração em Enfermagem, para obtenção do título de especialista em Estomaterapia, novembro de 2011.

Artigo recebido em: 14/03/2014 - Aceito para publicação em: 09/12/2014
} 


\section{RESUMEN}

Estudio cuantitativo transversal, para verificar la prevalencia de cateterismo urinario y caracterizar los recién-nacidos (RN's) internados en Unidad de Terapia Intensiva Neonatal (Neonatal UTI) que necesitan este procedimiento. Fueron evaluados prontuarios de 128 recién-nacidos admitidos entre enero de 2008 y diciembre de 2010 en la Unidad de Terapia Intensiva Neonatal de un hospital del Distrito Federal especializado en parto de alto riesgo. Los datos fueron analizados por medio del programa SPSS 11.5 for Windows. Fueron utilizados la prueba de Chi-cuadrado de Mantel-Haenszel con intervalo de confianza del $95 \%$ y la prueba t de Student, siendo considerados valores significativos $p<0,05$. Fue encontrada prevalencia de uso de catéter urinario de 15,6\% en la muestra estudiada. El principal motivo para la indicación del catéter fue la oliguria con el objetivo de control del débito urinario. Catéteres urinarios en Neonatal UTI están asociados a una mayor morbilidad relacionada a un mayor riesgo para sepsis y óbito.

DESCRIPTORES: Cateterismo urinario. Recién-nacido. Prevalencia. Enfermería neonatal.

\section{INTRODUÇÃO}

O Centers for Disease Control and Prevention (CDC) define cateter urinário $(\mathrm{CU})$ como um tubo de drenagem inserido na bexiga através da uretra, conectado ou não a um sistema de coleta fechado para drenagem da urina ${ }^{1}$. Os cateteres externos são formados por um invólucro de látex que se adere sobre o pênis, drenando a urina por um tubo acoplado ao látex ${ }^{1}$. Os CUs podem, ainda, ser introduzidos através de estomas confeccionados em casos de impossibilidade de drenagem da urina pela via natural.

O cateterismo urinário é um procedimento invasivo caracterizado pela introdução de um cateter até a bexiga através da uretra ou de um estoma, objetivando a drenagem da urina. Pode ser classificado como sendo de permanência — quando o cateter é mantido no trato genitourinário, enquanto permanecer a condição clínica que indicou o procedimento - , ou de alívio - quando o cateter é inserido e retirado logo após a drenagem da urina ${ }^{2}$. O cateterismo de alívio pode ser classificado, ainda, como intermitente, quando inserido a espaços determinados de tempo para esvaziamento da bexiga. No caso de cateterização urinária de permanência, ela pode ser de curta duração (até 7 dias), de duração intermediária (de 7 a 30 dias) e de longa duração (superior a 1 mês) $)^{3}$.

De acordo com a Agência Nacional de Vigilância Sanitária (ANVISA), o CU está associado a um risco de mortalidade três vezes maior nos hospitais e casas de repouso ${ }^{4}$. O uso de cateter vesical de permanência é comum em várias situações clínicas, sendo que a permanência do $\mathrm{CU}$ aumenta em $5 \%$ o risco de aparecimento de bacteriúria ${ }^{5}$, além de diversas outras complicações.

Diversos estudos têm sido realizados em relação aos CUs, mostrando sua incidência, complicações e relações com a ocorrência de infecções nosocomiais, especialmente relacionadas ao trato urinário ${ }^{6-8}$. Entretanto, os estudos realizados são voltados para o paciente adulto ou pediátrico ${ }^{9}$, pouco se conhecendo em relação ao recém-nascido (RN) que necessita fazer uso desse dispositivo.

A neonatologia abrange um período de vida relativamente curto, referindo-se aos primeiros 28 dias de vida pós-nascimento ${ }^{10}$. Apesar de curto, esse é um período extremamente crítico e instável, exigindo estudos voltados para essa população e profissionais cada vez mais especializados e preparados para lidar com as especificidades e fragilidades do RN. Ao nascimento, o RN passa, abruptamente, de um ambiente aconchegante - o útero materno - para um outro extremamente agressivo - o externo. Isso é ainda mais crítico no $\mathrm{RN}$ prematuro, cujo nascimento ocorre antes de 37 semanas gestacionais e frequentemente inicia sua vida em uma Unidade de Terapia Intensiva (UTI) repleta de estímulos intensos e estressantes ${ }^{11}$.

Em se tratando do paciente $\mathrm{RN}$, os cuidados e a abordagem devem ser diferenciados em virtude da fragilidade e das especificidades anatomofisiológicas. Atualmente, pouco se conhece acerca do RN em UTI neonatal que faça uso de CU, o que dificulta a implementação de cuidados baseados em evidências científicas. Nem mesmo se conhece a prevalência de cateterização urinária em $\mathrm{RNs}$ internados em UTI neonatal e os motivos que levam a tal procedimento. Portanto, o presente estudo teve como objetivo caracterizar o RN em UTI neonatal que necessita de cateterismo urinário em algum momento da internação, identificando a prevalência, as indicações terapêuticas para o procedimento, tempo e tipo de cateterização, fatores de comorbidade associados e complicações relacionadas ao procedimento. 


\section{MÉTODOS}

Trata-se de estudo transversal descritivo tendo como base a epidemiologia. Nessa modalidade de investigação, "causa"e "efeito" são detectados simultaneamente. Somente na análise dos dados é que se permite identificar os grupos de interesse, os “expostos", os "não expostos', os “doentes" e os "sadios"12. O estudo foi realizado em um hospital público terciário especializado em atenção materno-infantil em Brasília (DF). A amostra deste estudo foi selecionada a partir do total de pacientes internados entre janeiro de 2008 e dezembro de 2010 na UTI Neonatal do referido hospital, onde foram encontradas um total de 887 internações no período definido. Destas, selecionaram-se, aleatoriamente, 350 prontuários por meio do aplicativo Microsoft ${ }^{\circledR}$ Excel 2003. Dos 350 prontuários que deveriam ser pesquisados, foram localizados apenas $171 \mathrm{e}$, destes, 128 atendiam aos critérios de inclusão: pacientes com menos de 28 dias de vida no momento da primeira internação em Unidade Neonatal; pacientes que tinham completado a terapêutica na Unidade Neonatal do hospital de estudo; pacientes admitidos de outras Unidades Neonatais com menos de 36 horas de internação; e prontuários completos. Prontuários com dados incompletos; pacientes com mais de 28 dias de vida na primeira internação; pacientes admitidos de outros hospitais com mais de 36 horas de internação e aqueles que haviam sido transferidos antes da conclusão da terapêutica foram excluídos.

Os dados secundários foram coletados nos prontuários a partir das evoluções médicas e de enfermagem, anotações de enfermagem e prescrições médicas, utilizando-se instrumento específico elaborado pela pesquisadora. Para fins de homogeneização da amostra e para possibilitar o tratamento dos dados, os diagnósticos médicos foram relacionados por sistemas orgânicos e separados em diagnósticos precoces obtidos no momento da internação e diagnósticos tardios em decorrência de complicações durante a internação, tendo como parâmetro a Classificação Internacional de Doenças e Problemas Relacionados à Saúde (CID-10) ${ }^{13}$ Diagnósticos relacionados a malformações congênitas não foram contabilizados, já que estas foram classificadas em um grupo próprio de comparação.

Os dados foram analisados por meio do programa estatístico Statistical Package for the Social Sciences (SPSS) 11.5 for Windows ${ }^{\circledR 14}$. Foi verificada a prevalência de cateteres urinários em RNs internados em UTI Neonatal, e, como variáveis independentes, foram analisados o tipo, o tempo e o motivo da cateterização e comorbidades associadas. Para a análise de possíveis associações entre variáveis, foi utilizado o teste do $\chi^{2}$ de Mantel-Haenszel para variáveis categóricas, sendo considerado intervalo de confiança de 95\% (IC95\%), e o teste $t$ de Student para variáveis numéricas, sendo considerados valores significantes $\mathrm{p}<0,05$.

Este estudo foi avaliado e aprovado pelo Comitê de Ética em Pesquisa da Fundação de Ensino e Pesquisa em Ciências da Saúde do Distrito Federal (FEPECS), sob o protocolo no 048/11, tendo sido dispensado o Termo de Consentimento Livre e Esclarecido por se tratar de pesquisa documental.

\section{RESULTADOS}

A amostra analisada caracterizou-se por um total de 128 RNs, dos quais 75 (58,6\%) eram do sexo masculino e 53 $(41,4 \%)$, do sexo feminino. A média de idade gestacional ao nascimento foi de 30 semanas e 6 dias, sendo a maior prevalência de $\mathrm{RNs}$ nascidos de 28 semanas, com mínimo de 24 semanas e máximo de 42 semanas. Quanto ao tipo de parto, $50(39,1 \%)$ nasceram de parto normal, enquanto 75 (58,6\%) nasceram de parto cesáreo. Os RNs tiveram um Apgar no $1^{\circ}$ minuto com média de 6,45, enquanto o Apgar no $5^{\circ}$ minuto teve média de 8,29. A média de peso ao nascimento foi de $1.589,66 \mathrm{~g}$, sendo que o valor mais prevalente foi de $750 \mathrm{~g}$.

Os diagnósticos identificados na amostra durante a internação são apresentados na Figura 1 e estratificados em precoces e tardios.

Quanto aos agentes infecciosos, a maior prevalência foi de gram-positivos (21,5\%), embora não tenha sido possível identificar o agente causador em $64,5 \%$ dos RNs identificados com sepse. Dos RNs investigados, 15,6\% foram submetidos a algum procedimento cirúrgico, com maior prevalência para laparatomias $(6,3 \%)$, seguidas de herniorrafia $(1,6 \%)$, correção total de gastrosquise $(2,3 \%)$, derivação ventriculoperitoneal $(1,6 \%)$ e outros $(3,8 \%)$.

A prevalência de malformação congênita na amostra foi de 22,7\%, abrangendo malformações cardíacas (septo e grandes artérias), malformação de face, esôfago, traqueia e pulmão, malformação de membros e esqueleto, gastrosquise, 


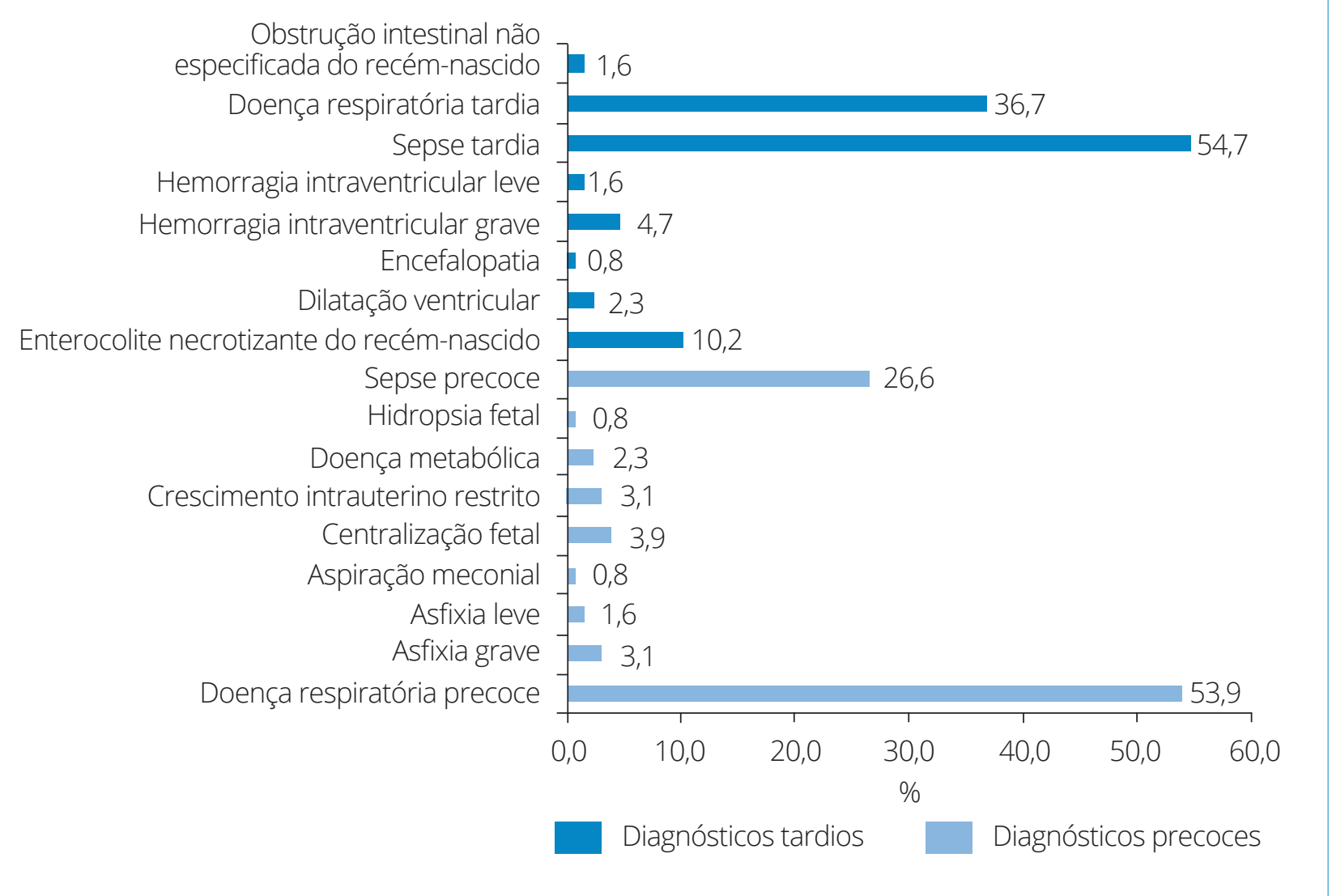

Figura 1. Distribuição dos diagnósticos precoces e tardios encontrados durante a internação em Unidade de Terapia Intensiva Neonatal de um hospital materno-infantil do Distrito Federal de 2008 a 2010.

malformações intestinais, anencefalia e microcefalia. $\mathrm{O}$ tempo médio de internação em UTI Neonatal para a amostra estudada foi de aproximadamente 30 dias, com o máximo de 141 dias e mínimo de 1 dia de internação, sendo que a taxa de óbito foi de $27,3 \%$.

A prevalência de uso de CU na amostra foi de 15,6\%, ou seja, $20 \mathrm{RNs}$ foram cateterizados em algum momento durante a internação. A média de tempo de cateterização urinária nesses RNs, em dias, foi de 4,65 ( $\pm 6,51)$, e a média de trocas ao longo do período de cateterização foi de $0,6( \pm 1,35)$, sendo o máximo de trocas encontradas de 5 vezes.

Quando avaliado o tipo de cateter urinário mais utilizado em UTI Neonatal, foram encontradas 3 ocorrências com cateter foley (15\%) e 4 com cateter de nelaton (20\%). Entretanto, em 13 casos (65\%), não foi relatado o procedimento, bem como o tipo de cateter utilizado. $\mathrm{O}$ mesmo ocorreu em relação ao tipo de material do cateter, tendo sido encontrados 2 relatos de cateter siliconado (10\%) e 18 relatos não informados (90\%). Quanto ao tamanho do cateter, foram utilizados os calibres 4 Fr para
RNs entre 28 e 30 semanas de idade gestacional; 6 Fr para RNs entre 34 e 38 semanas de idade gestacional e 8 e 10 Fr para RNs de 39 a 42 semanas de idade gestacional. Os calibres mais frequentes foram 4 e $6 \mathrm{Fr}$, ressaltando-se que, em 12 (60\%) casos, não foi informado o calibre do cateter utilizado. Quanto às condições clínicas que resultaram na indicação para o cateterismo vesical e retirada do cateter, bem como os objetivos do procedimento e os motivos da troca, os dados são apresentados na Figura 2.

Quanto às complicações relacionadas ao cateterismo, foi encontrado apenas um relato de piúria, um relato de edema genital e outro relato de hematúria, sendo que não foi possível identificar se estes dois últimos eventos eram relacionados ao uso do cateter ou à própria condição clínica dos RNs, que se apresentavam edemaciados e com diagnóstico de insuficiência renal aguda (IRA).

As características e diferenças estatísticas encontradas quando comparado o grupo de RNs que usou cateter vesical e aqueles que não utilizaram cateter vesical durante a internação estão apresentadas nas Tabelas 1 e 2 . 


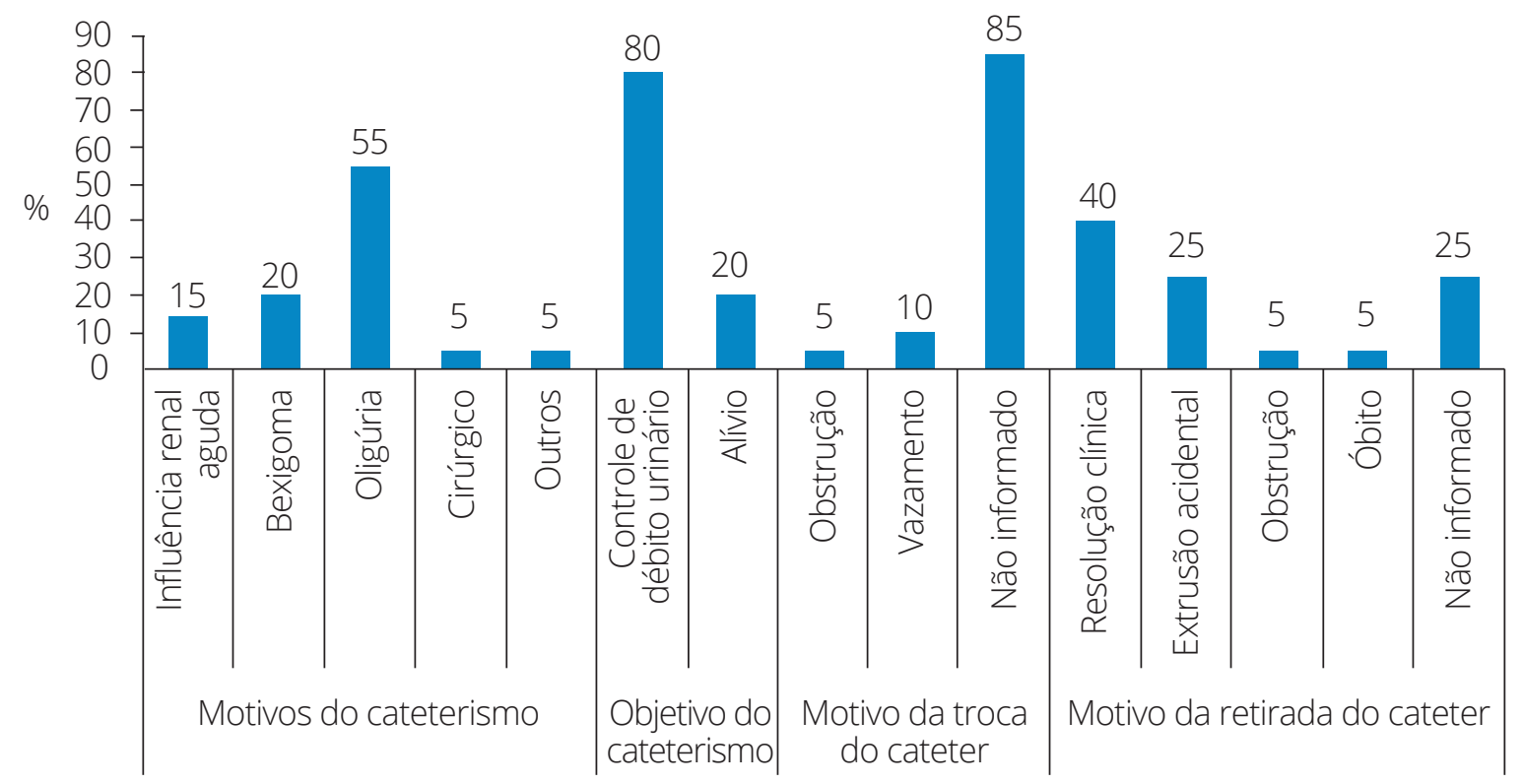

Figura 2. Características da cateterização urinária por motivos e objetivos do procedimento, motivos da troca e retirada do cateter em recém-nascidos internados em Unidade de Terapia Intensiva Neonatal de um hospital materno-infantil do Distrito Federal entre 2008 e 2010.

Tabela 1. Comparação das variáveis independentes entre recém-nascidos que utilizaram cateter urinário e recém-nascidos que não utilizaram cateter urinário, considerando-se média e desvio padrão, quando foram internados em Unidade de Terapia Intensiva Neonatal de um hospital materno-infantil do Distrito Federal entre 2008 e 2010.

\begin{tabular}{|c|c|c|c|}
\hline \multirow{3}{*}{ Variável independente } & Com cateter & Sem cateter & \multirow{3}{*}{$\mathrm{p}$} \\
\hline & $\mathrm{n}=20$ & $n=108$ & \\
\hline & Média ( \pm desvio padrão) & Média ( \pm desvio padrão) & \\
\hline Idade gestacional (em semanas) & $32,9( \pm 4,48)$ & $31,1( \pm 4,22)$ & 0,088 \\
\hline Peso ao nascimento (em gramas) & $1.904,21( \pm 1031,66)$ & $1.534,32( \pm 828,41)$ & 0,087 \\
\hline Apgar no $1^{\circ}$ minuto & $5,56( \pm 2,85)$ & $6,61( \pm 1,99)$ & 0,055 \\
\hline Apgar no $5^{\circ}$ minuto & $7,78( \pm 2,1)$ & $8,38( \pm 1,2)$ & 0,086 \\
\hline $\begin{array}{l}\text { Tempo de uso de dissecção venosa } \\
\text { (em dias) }\end{array}$ & $24,69( \pm 23,18)$ & $11,11( \pm 8)$ & $0,026 *$ \\
\hline Tempo de uso de PICC (em dias) & $24,56( \pm 18,9)$ & $21,15( \pm 12,3)$ & 0,503 \\
\hline $\begin{array}{l}\text { Tempo de uso de nutrição parenteral } \\
\text { (em dias) }\end{array}$ & $26,76( \pm 14,88)$ & $17( \pm 12,2)$ & 0,001 * \\
\hline $\begin{array}{l}\text { Tempo de uso de cateterismo } \\
\text { umbilical (em dias) }\end{array}$ & $7,29( \pm 6,52)$ & $8,17( \pm 6,36)$ & 0,635 \\
\hline $\begin{array}{l}\text { Tempo de uso de ventilação } \\
\text { mecânica (em dias) }\end{array}$ & $36,26( \pm 39,56)$ & $15( \pm 18,54)$ & 0,001 * \\
\hline $\begin{array}{l}\text { Tempo de uso de ventilação não } \\
\text { invasiva (em dias) }\end{array}$ & $8,25( \pm 7)$ & $13,5( \pm 13,17)$ & 0,124 \\
\hline Número de transfusões & $7( \pm 4,33)$ & $4,75( \pm 3,32)$ & $0,022^{\star}$ \\
\hline Tempo de hospitalização (em dias) & $44,65( \pm 36,89)$ & $27,75( \pm 25,48)$ & $0,013 *$ \\
\hline
\end{tabular}

*Valores significantes $p<0,05$.

PICC: cateter central de inserção periférica. 
Tabela 2. Comparação das variáveis independentes entre recém-nascidos que utilizaram cateter urinário e recém-nascidos que não utilizaram cateter urinário, por número e porcentagem do evento, quando internados em Unidade de Terapia Intensiva Neonatal de um hospital materno-infantil do Distrito Federal entre 2008 e 2010.

\begin{tabular}{|c|c|c|c|c|c|}
\hline Variável independente & $\begin{array}{c}\text { Com cateter } \\
\mathrm{n}(\%)\end{array}$ & $\begin{array}{l}\text { Sem cateter } \\
\mathrm{n}(\%)\end{array}$ & RR & IC95\% & $\mathrm{p}$ \\
\hline Parto cesáreo & $12(63,2)$ & $63(59,4)$ & 1 & $0,7-1,5$ & 0,8 \\
\hline Sexo masculino & $9(45)$ & $66(61,1)$ & 0,7 & $0,4-1,2$ & 0,2 \\
\hline Uso de dissecção venosa & $15(75)$ & $16(14,8)$ & 5 & $3-8,5$ & 0,000 * \\
\hline Uso de acesso venoso periférico & $18(90)$ & $86(79,6)$ & 1,13 & $0,9-1,3$ & 0,3 \\
\hline Uso de PICC & $9(45)$ & $40(37)$ & 1,2 & $0,7-2$ & 0,6 \\
\hline Uso de nutrição parenteral & $17(85)$ & $78(72,2)$ & 1,1 & $0,9-1,4$ & 0,2 \\
\hline Uso de cateter umbilical & $14(70)$ & $76(70,4)$ & 0,99 & $0,7-1,3$ & 1 \\
\hline Uso de ventilação mecânica & $19(95)$ & $69(63,9)$ & 1,48 & $1,2-1,7$ & $0,004 *$ \\
\hline Uso de oxigenoterapia não invasiva & $16(80)$ & $93(86,9)$ & 0,9 & $0,7-1,1$ & 0,4 \\
\hline Infecção durante internação & $18(90)$ & $89(82,4)$ & 1 & $0,9-1,2$ & 0,5 \\
\hline Diagnóstico de sepse tardia & $17(85)$ & $53(49,1)$ & 1,7 & $1,3-2,2$ & $0,003^{*}$ \\
\hline Diagnóstico de doença respiratória tardia & $9(47,4)$ & $38(35,8)$ & 1,3 & $0,7-2,2$ & 0,4 \\
\hline $\begin{array}{l}\text { Quatro ou mais esquemas de } \\
\text { antibioticoterapia }\end{array}$ & $4(20)$ & $2(1,9)$ & 3,3 & $1,8-5,8$ & 0,006 * \\
\hline Uso de anfotericina $\mathrm{B}$ & $10(50)$ & $15(13,9)$ & 3,6 & $1,8-6,8$ & 0,001 * \\
\hline Presença de malformação congênita & $11(55)$ & $18(16,7)$ & 3,3 & $1,8-5,8$ & 0,001 * \\
\hline Procedimento cirúrgico & $10(50)$ & $10(9,3)$ & 5,4 & $2,5-11,2$ & $0,000 *$ \\
\hline Hemoterapia & $18(90)$ & $60(55,6)$ & 1,6 & $1,2-2$ & $0,005^{*}$ \\
\hline Uso de drogas vasoativas & $19(95)$ & $39(36,1)$ & 2,6 & $2-3,4$ & $0,000^{*}$ \\
\hline Óbito & $10(50)$ & $25(23,1)$ & 2,1 & $1,2-3,7$ & $0,026 *$ \\
\hline
\end{tabular}

*Valores significantes $p<0,05$.

RR: risco relativo; IC95\%: intervalo de confiança de 95\%; PICC: cateter central de inserção periférica.

\section{DISCUSSÃO}

A prevalência do uso de CU no grupo estudado foi de $15,6 \%(n=20)$, valor de acordo com o encontrado na literatura para adultos, cuja prevalência de CUs entre pacientes adultos internados em diversas clínicas norte-americanas varia de 7 a $25 \% \%^{1,15}$, sendo que, no Brasil, há relato de prevalência de $10^{6}$ a $14 \%{ }^{16}$. No entanto, é necessário ressaltar que a população de estudo neste trabalho foi restrita à UTI, onde o uso de CU é expressivo, frequentemente em virtude da gravidade clínica dos pacientes ${ }^{17}$.
A cateterização urinária, seja ela contínua ou de alívio, está corretamente indicada nos casos de: retenção de urina; alívio da obstrução do trato urinário; perioperatório de cirurgias urológicas ou de estruturas contíguas, cirurgias demoradas ou necessidade de controle do débito urinário no intraoperatório; controle de débito urinário em pacientes em estado crítico; promoção de drenagem urinária nos pacientes com disfunção neurogênica ou retenção urinária; impedimento do extravasamento urinário nos pacientes com úlceras de pressão em estágio III ou $\mathrm{IV}^{18}$. A orientação do $\mathrm{CDC}^{1}$ é de que o $\mathrm{CU}$ seja 
utilizado nos casos indicados e retirado o mais precocemente possível, ou até mesmo que a cateterização contínua seja substituída, em alguns casos, por alternativas terapêuticas, como o cateter externo em homens incontinentes ou o cateterismo intermitente nas retenções urinárias pós-cirúrgicas. Nesses casos, o cateter deve ser inserido e retirado logo após a drenagem da urina.

Entre crianças, o uso do cateter vesical é mais comumente citado nos casos de mielomeningocele, bexiga neurogênica e para coletas de amostra de urinocultura ${ }^{1}$. Na amostra analisada, o principal objetivo para a cateterização urinária foi o controle do débito urinário (80\%; $\mathrm{n}=16)$, seguido da necessidade de drenagem de urina em virtude de retenção urinária $(20 \% ; n=4)$ evidenciada por bexigoma. A principal indicação clínica foi a oligúria $(55 \% ; n=11)$ devido à sepse tardia ou em virtude de pós-operatório imediato. A ocorrência de malformações congênitas foi encontrada como outro fator para a cateterização urinária, independentemente do tipo de malformação, já que, em nenhum caso, houve relação direta com malformações ou alterações específicas do trato genitourinário. A ocorrência de malformação congênita aumentou o risco de $\mathrm{CU}$ em três vezes. Adicionalmente, procedimentos cirúrgicos também parecem estar relacionados, mesmo que indiretamente, à necessidade de cateterização urinária em UTI Neonatal, aumentando esse risco em cinco vezes. Foi observado que $30 \%(n=6)$ das cateterizações ocorreram em virtude de retenção urinária ou descompensação hemodinâmica no pós-operatório imediato, uma vez que o procedimento cirúrgico no neonato, já tão fragilizado, provoca estresse fisiológico, levando, muitas vezes, à desidratação ou choque com consequente oligúria, o que exige um rígido controle do débito urinário ${ }^{19}$.

$\mathrm{Na}$ literatura, o tempo médio de cateterização urinária refere-se ao paciente adulto internado, variando de 48 horas a 7,9 dias $^{20}$. Neste estudo, a média de tempo de cateterização no $\mathrm{RN}$ foi equivalente ao paciente adulto, com média de 4,65 dias, sendo o máximo de 29 dias e o mínimo de 1 dia.É importante ressaltar que todas as cateterizações foram de permanência, ou seja, o cateter permaneceu no trato genitourinário por pelo menos 24 horas. Por outro lado, há viés na amostra, visto que apenas $1 \mathrm{RN}$ ficou 29 dias com cateter vesical de permanência, elevando a média de tempo e o desvio padrão, enquanto os demais permaneceram entre 1 e 5 dias cateterizados. O mesmo ocorreu com o número de trocas: um RN necessitou de cinco trocas do cateter vesical de permanência ao longo do tratamento, enquanto os demais não necessitaram de trocas ou chegaram a ter apenas uma troca do cateter.
O tempo de permanência do cateter deve ser avaliado segundo a indicação clínica da cateterização, devendo-se retirar o cateter tão logo cesse essa condição. Quanto menor o tempo de permanência do cateter, menores os riscos de complicações associadas. Estudos com o paciente adulto cateterizado durante procedimento cirúrgico mostram tempo tolerável de cateterização de até 48 horas, caso não haja nenhuma condição que contraindique sua retirada ${ }^{16}$. Nesta amostra, quando a cateterização foi indicada no pós-cirúrgico, o cateter permaneceu por no máximo 24 horas. Por outro lado, quando o motivo para cateterização foi a presença de retenção urinária, o cateter permaneceu 1, 3, 6 e 29 dias. Segundo a European Association of Urology Nurses, a retenção urinária é uma das condições não neurogênicas para indicação de cateterismo de alívio como alternativa ao cateterismo de demora ${ }^{21}$.

Foi verificada, ainda, maior prevalência do uso de cateter de nelaton $(20 \% ; \mathrm{n}=4)$ quando comparado ao uso de cateter foley (15\%; n=3). Entretanto, a quantidade de casos omissos por falta de anotação foi bastante elevada $(65 \%$; n=13), não sendo possível verificar os principais tipos de CUs utilizados em UTI Neonatal. Por outro lado, visto que a extrusão acidental ocorreu em $25 \%(\mathrm{n}=5)$ dos casos, é possível deduzir que o uso do cateter de nelaton para cateterização de permanência foi mais recorrente, já que esse tipo de cateter não possui sistema adequado de fixação, o que facilita sua saída acidental.

É fundamental ressaltar que o tipo de cateter deve ser adequado para cada indicação clínica e objetivos do procedimento. Neste estudo, embora o cateterismo de alívio tenha sido o objetivo do procedimento em $20 \%$ ( $n=4)$ dos casos, ainda assim o cateter de nelaton permaneceu por pelo menos 24 horas. Já no manejo da oligúria, o cateter de nelaton foi informado em pelo menos 2 casos e permaneceu por até 14 dias, não se contando as trocas do cateter ao longo desse período. Em virtude dos riscos, a permanência desse tipo de cateter no trato genitourinário é inapropriada e desaconselhada ${ }^{22}$. Outro agravante é que o cateter de nelaton não possui conector apropriado que garanta um sistema de drenagem ocluído e estéril durante toda a terapêutica.

Por outro lado, uma das dificuldades no uso de CU em neonatos está no tamanho adequado do dispositivo. Os cateteres foley de menor calibre disponíveis atualmente no mercado brasileiro são de 6 Fr. Entretanto, para o RN prematuro de muito baixo peso, são requeridos calibres menores. Não há um consenso na literatura sobre o calibre adequado do $\mathrm{CU}$ para o paciente adulto, pediátrico ou 
neonato, e as informações são divergentes. Uma guideline europeia indica cateteres de $5 \mathrm{Fr}$ para pacientes menores de 6 meses, enquanto os de 6 e 8 Fr devem ser utilizados apenas em crianças com mais de 6 meses $^{21}$. Outra guideline $e^{23}$ relaciona o calibre do cateter com o peso do paciente, indicando o uso de cateter umbilical para cateterização urinária: calibre de 3,5 Fr para neonatos com menos de $1.200 \mathrm{~g}$; calibre de 5 Fr para nonatos entre $1.200-1.500 \mathrm{~g}$ e calibres de 5 e 6 Fr para neonatos entre 1.500-2.500 g. Nessa faixa de peso e acima, já se considera adequado o uso de cateter foley $6 \mathrm{Fr}$, preferencialmente de silicone. Um terceiro autor considera adequado o uso de cateter de nelaton 4 Fr para neonatos menores, sem fazer alusão à idade ou ao peso ${ }^{24}$. Entretanto, estudos controlados devem ser propostos a fim de se verificar a segurança desses dispositivos na cateterização urinária de neonatos.

$\mathrm{Na}$ amostra estudada, não foi possível verificar o material do cateter, já que não houve informação em 90\% ( $\mathrm{n}=18$ ) dos casos. Quanto ao calibre, foi possível relacioná-lo ao peso do RN. Calibres de 4 Fr foram utilizados em neonatos entre $930-1.545 \mathrm{~g}$. Já cateteres de calibre de 6 Fr foram usados em RNs de 1.640-2.360 g. Cateteres de 8 e $10 \mathrm{Fr}$ foram utilizados em neonatos de 3.450-4.040 g. Apesar de não haver consenso na literatura quanto ao calibre adequado do cateter, é orientado o uso do menor calibre disponível e que garanta a melhor drenagem do fluído. Provavelmente, um cateter de calibre acima de 6 Fr para um RN seja desnecessário para garantir a drenagem adequada de urina. Por outro lado, há que se destacar o alto número de casos omissos (60\%; $n=12)$, impossibilitando um conhecimento real acerca do tamanho do cateter usado em neonatos.

O material do cateter, bem como o calibre adequado para cada paciente, assume importância clínica na prevenção de complicações associadas à cateterização vesical, como a uretrite e o traumatismo uretra ${ }^{15}$. Estudos clínicos têm identificado menor incidência de uretrite com cateter de silicone quando comparado ao cateter de láte $\mathrm{x}^{20}$. $\mathrm{O}$ trauma de uretra pode ser minimizado por meio dos seguintes cuidados: uso de um cateter de tamanho apropriado, lubrificação adequada do cateter com uso de solução hidrossolúvel durante a inserção e inserção do cateter por uma distância suficiente dentro da bexiga para evitar trauma dos tecidos uretrais quando o balão de retenção do cateter é insuflado ${ }^{18}$. Outras complicações que podem estar relacionadas ao cateterismo urinário referem-se a espasmos vesicais, estenoses uretrais, necrose por pressão e infecção do trato urinário (ITU) ${ }^{18,22}$.
Quanto às complicações relacionadas ao cateterismo vesical, não foi possível fazer a correlação na amostra estudada. Os relatos de edema genital e hematúria poderiam estar relacionados à condição clínica dos RNs, que apresentavam IRA. Entretanto, a ocorrência de hematúria após a instrumentação do trato urinário pode ocorrer no caso de trauma uretral ${ }^{22,25}$.Já o relato de secreção purulenta no meato uretral, encontrado no RN com maior tempo de cateterização urinária (29 dias), muitas vezes apresenta-se enquanto sinal de ITU e refere-se à colonização bacteriana do cateter, que ocorre por aderência a sua face interna e ascensão até o trato genitourinário ${ }^{22}$.

Embora não tenham sido identificadas complicações diretamente relacionadas ao uso de cateter vesical no $\mathrm{RN}$ internado em UTI Neonatal, identificamos que esse paciente, já fragilizado e imunodeprimido, possui comorbidades associadas que podem agravar o seu estado clínico, sendo o dispositivo urinário um fator de risco adicional, o que exige do enfermeiro atenção para os cuidados com a inserção e manutenção do dispositivo a fim de evitar complicações associadas.

A ITU constitui um dos principais tipos de infecção hospitalar, sendo a presença de $\mathrm{CU}$ o principal fator de risco ${ }^{1}$. Das infecções hospitalares, $40 \%$ estão localizadas no trato urinário, sendo que $60 \%$ são relacionadas ao cateter de permanência ${ }^{15}$. Entretanto, não foi encontrado relato de ITU na amostra estudada. Na verdade, os dados mostraram que é a ocorrência de sepse que leva à indicação da cateterização urinária pela necessidade de controle do débito urinário no paciente crítico. No RN, os mecanismos de evolução da sepse tardia resultam em choque, hipoperfusão e consequente oligúria ${ }^{26}$, um dos principais motivos para cateterização na amostra deste estudo. Além disso, no grupo de RNs estudados, a cateterização urinária durou, em média, 4,6 dias, não havendo tempo suficiente para a ocorrência de bacteriúria que, segundo a literatura, ocorre entre 7 e 14 dias após a instalação do cateter ${ }^{18}$.

O uso de cateter urinário esteve também, no presente estudo, significativamente associado à presença de comorbidades ou condições clínicas e terapêuticas críticas, como existência de dissecção venosa, tempo prolongado de nutrição parenteral e ventilação mecânica, necessidade de hemotransfusões, uso de drogas vasoativas, necessidade de mais esquemas de antibióticos e uso de anfotericina B. Todas essas medidas terapêuticas muitas vezes são aplicadas no controle e reversão da sepse $\operatorname{tardia}^{26}$. Corroborando esses dados, observou-se no presente estudo que o número de óbitos entre o grupo 
de $\mathrm{RN}$ s que usou cateter vesical contínuo foi 2 vezes maior $(\mathrm{p}=0,026)$ que entre o grupo de $\mathrm{RN}$ s que não necessitou do uso desse dispositivo (Tabela 2). Além disso, o uso do cateter vesical de permanência foi relacionado a um aumento no tempo de internação de até 20 dias, o que revela um paciente mais grave, mais fragilizado e mais exposto aos riscos de procedimentos invasivos e estressantes.

Uma das limitações que este estudo apresenta é ter sido realizado em um único centro, o que pode dificultar a generalização dos achados, especialmente porque o hospital onde ocorreu a coleta de dados é referência em assistência infantil, recebendo os casos mais graves. Além disso, a frequência de utilização do CU, suas indicações e tempo de permanência bem como a forma de registro variam com as características dos hospitais, da população assistida e dos processos de trabalho. Vale ressaltar, também, que registros de dados em prontuários não são anotados para a finalidade de pesquisa, o que pode comprometer a qualidade dos registros. Isso fica evidente pela quantidade de anotações incompletas ou dados não relatados. Apesar das limitações, este estudo traz uma nova perspectiva de investigação para o estomaterapeuta, cujas contribuições podem auxiliar no avanço da assistência ao RN de alto risco.

\section{CONCLUSÃO}

A partir dos dados analisados e das informações geradas, pode-se concluir que o uso do CU de permanência está associado a uma maior morbidade em UTI Neonatal. Embora não exista relação de causa-efeito, o cateterismo urinário foi relacionado a um risco de sepse e de óbito aumentados, mostrando a maior gravidade do RN que faz uso do dispositivo durante o período de internação em UTI Neonatal.

Este estudo encontrou prevalência de uso de $\mathrm{CU}$ em RNs dentro daquela esperada para o paciente adulto, correspondente a 15,6\%. Entretanto, é fundamental observar que os estudos utilizados para fins de comparação de prevalência de CUs em adultos abrangem todas as clínicas de internação e não apenas o paciente crítico em cuidados intensivos, como foi feito nesta pesquisa.

$\mathrm{Na}$ amostra estudada, as principais indicações clínicas para a cateterização urinária em RNs na UTI Neonatal foram o procedimento cirúrgico e a sepse, com o objetivo de controle de débito urinário ou alívio vesical. O principal tipo de cateter utilizado foi o cateter de nelaton, mesmo para cateterizações urinárias de permanência. Por outro lado, a cateterização de permanência foi utilizada de forma indiscriminada, mesmo quando o objetivo era o esvaziamento da bexiga. A média de permanência do cateter em dias não parece ser suficiente para ocasionar complicações em UTI Neonatal. No entanto, estudos prospectivos poderiam avaliar essa correlação com maior propriedade. Não existe consenso na literatura para o calibre de cateter mais adequado à população neonatal, além da escassez de dispositivos voltados às necessidades específicas do neonato prematuro extremo. Sendo assim, em alguns casos, torna-se necessária a adaptação de outros dispositivos pela equipe de enfermagem. A falta de dados na literatura, de protocolos e de estudos sistematizados voltados para a o uso de CU na população neonatal traz prejuízos à qualidade da assistência de enfermagem, fazendo com que o profissional tenha que lançar mão de recursos baseados no empirismo. Além disso, a escassez de estudos relacionados à cateterização urinária no RN dificultou a comparação dos resultados encontrados.

A falta de anotações de enfermagem em relação ao procedimento resultou em dados omissos, que impediram correlações mais efetivas, como o calibre do cateter, a idade e o peso do RN. O registro dos procedimentos pelo enfermeiro assume importância para a avaliação dos cuidados e o desenvolvimento de estudos epidemiológicos que possam oferecer arcabouço científico para uma assistência baseada em evidências. As características do cateter utilizado (calibre, tipo etc.) e as complicações associadas devem ser relatadas a fim de favorecer uma assistência direcionada e racionalizada. Estudos prospectivos devem ser propostos para se verificarem os cuidados do profissional em relação ao procedimento, a escolha correta do calibre e o custo-benefício quanto ao material de escolha do cateter. Por outro lado, destaca-se também a necessidade de desenvolverem dispositivos urinários específicos para o neonato.

A presente investigação permitiu traçar um perfil epidemiológico do $\mathrm{RN}$ que necessita de cateterização urinária em uma UTI, contribuindo para uma reflexão da prática profissional quanto à indicação da cateterização urinária no $\mathrm{RN}$ bem como ao tipo de cateter escolhido para cada procedimento, levando-se em consideração as indicações clínicas e os objetivos do procedimento. O trabalho permitiu também, indiretamente, uma reflexão para a prática do enfermeiro, ressaltando a importância do pensamento crítico na execução de procedimentos e intervenções no RN internado em UTI Neonatal, considerando-se sua fragilidade e gravidade clínica. 


\section{REFERÊNCIAS}

1. Centers for Disease Control and Prevention. Guidelines for Prevention of Catheter - Associated Urinary Tract Infections. USA: Department of Health and Human Services; 2009.

2. Australian and New Zealand Urological Nurses Society Inc. [Internet]. Catheterisation Clinical Guidelines. April 2013 [cited 2014 Dec 30]. Available from: http://www.anzuns. org/wp-content/uploads/2011/12/ANZUNS-Catheterisationfinal-Document-October-20131.pdf

3. Maki DG, Tambyah PA. Engineering out the risk of infection with urinary catheters. Emerg Infect Dis. 2001;7(2):1-6.

4. Agência Nacional de Vigilância Sanitária. Curso básico de controle de infecção hospitalar - Caderno B - Principais síndromes infecciosas hospitalares. Brasil: Ministério da Saúde; 2000

5. Stamm AMNF, Coutinho MSSA. Infecção do trato urinário relacionada a cateter vesical de demora: incidência e fatores de risco. Rev Assoc Med Bras. 1999;45(1):27-33.

6. Lima LS, Araújo EC, Bezerra SMMS, Linhares FM, Lima AKA. Urinary tract infections focused on long-therm catheterization of patients in intensive therapy unit of Recife (PE), Brazil. Enfermería Global. 2007;11:1-10.

7. Dantas NM. Infecção do trato urinário. In: Oliveira AC, Albuquerque CP, Rocha LCM. Infecções hospitalares: abordagem, prevençäo e controle. Rio de Janeiro: Medsi; 1998.

8. Almeida MC, Simões MJS, Raddi MSG. Ocorrência de infecção urinária em pacientes de um hospital universitário. Rev Ciênc Farm Básica Apl. 2007;28(2):215-9.

9. Alves LC, Petrício JL, Garcia LGF, Souza ES, Ferreira EB. Incidência das infecções de corrente sanguínea, do trato urinário e pneumonia na unidade de terapia intensiva pediátrica de um hospital de ensino no ano de 2008/2009. Proceedings of the $61^{\circ}$ Congresso Brasileiro de Enfermagem; 2009 dez.; Fortaleza, Brasil. Trabalho 1474-1/3. p. 4645-7.

10. Brasil. Ministério da Saúde. Secretaria de Políticas de Saúde. Departamento de Atenção Básica. Saúde da criança: acompanhamento do crescimento e desenvolvimento infantil/Ministério da Saúde. Secretaria de Políticas de Saúde. Brasília: Ministério da Saúde; 2002.

11. Doré Júnior $S$, Imamura PEA, Figueira BBD, Fernandes GCV. Humanização e abordagem desenvolvimentista nos cuidados ao recém-nascido de muito baixo peso. In: Costa HPF, Marba ST, coordenadores. O recém-nascido de muito baixo peso. São Paulo: Atheneu; 2006. p. 85-102.

12. Pereira MG. Epidemiologia: teoria e prática. Rio de Janeiro: Guanabara Koogan; 2010.

13. Classificação Internacional de Doenças e Problemas Relacionados à Saúde [Internet].. Centro Colaborador da OMS para a Classificação de Doenças em Português-
CBCD; 2008 [cited 2011 Jun 12]. Available from: http://www. datasus.gov.br/cid10/v2008/cid10.htm

14. Statistical Package for the Social Sciences [Internet]. SPSS step-by-step. Tutorial: part 1. DataStep Development; 2004 [cited 2014 Dec 30]. Available from: www.datastep.com/ SPSSTutorial_1.pdf

15. Lenz LL. Cateterismo vesical: cuidados, complicações e medidas preventivas. ACM Arq Catarin Med. 2006;35(1):82-91.

16. Conterno LO, Lobo JA, Massom W. Uso excessivo do cateter vesical em pacientes internados em enfermarias de hospital universitário. Rev Esc Enferm USP. 2011;45(5):1089-96.

17. Juan LFV, Campos RM, González MCS. Incidencia de la infección nosocomial en la Unidad de Cuidados Intensivos del Hospital Clínico de Salamanca (1993-1994). Rev Clin Esp. 1996;196(5):281-8.

18. Smeltzer SC, Bare BG, Hinkle JL, Cheever KH. Tratado de enfermagem médico-cirúrgica. v. 3. $10^{a}$ edição. Rio de Janeiro: Guanabara Koogan; 2011.

19. Tamez RN, Silva MJP. Enfermagem na UTI Neonatal: assistência ao recém-nascido de alto risco. $3^{a}$ edição. Rio de Janeiro: Guanabara Koogan; 2006.

20. Schumm K, Lam TBL. Types of urethral catheters for management of short-term voiding problems in hospitalised adults. Cochrane Database Syst Rev. 2008;(2):CD004013.

21. European Association of Urology Nurses [Internet]. Urethral catheterization: male, female and paediatric intermittent catheterization. Section 2. 2006 [cited 2014 Dec 30]. Available from: http://www.uroweb.org/fileadmin/user_ upload/EAUN/EAUN1.pdf

22. Stamm AMNF, Forte DY, Sakamoto KS, Campos ML, Cipriano ZM. Cateterização vesical e infecção do trato urinário: estudo de 1092 casos. ACM Arq Catarin Med. 2006;35(2):72-7.

23. Scoble $M$, coordenador [Internet]. Indwelling urinary catheter - insertion and ongoing care. Clinical Guidelines (Nursing). The Royal Children's Hospital Melbourne. 2013 [cited 2014 Dec 30]. Available from: http://www.rch.org.au/ rchcpg/hospital_clinical_guideline_index/Indwelling_urinary_ catheter_insertion_and_ongoing_care/

24. Pellowe CM, Rogers J. Preventing healthcare-associated infections when using urinary catheters. Infant. 2007;3(4):150-1.

25. Elizondo AP, Gilbert J, Wearden ME, Lanclos D, Adams J. Elimination of bleeding associated with urinary catheterization in neonates. Neonatal Netw. 2001;20(6):25-34.

26. Fazio J. Moléstias infecciosas: sepse em neonatologia. In: Carvalho WB, Hirschhmeimer MR, Matsumoto T. Terapia intensiva pediátrica. $3^{a}$ edição. São Paulo: Atheneu; 2010. p. 914-37. 